\title{
LENGTH FOUR POLYNOMIAL AUTOMORPHISMS
}

\author{
SOORAJ KUTTYKRISHNAN
}

\begin{abstract}
We study the structure of length four polynomial automorphisms of $R[X, Y]$ when $R$ is a UFD. The results from this study are used to prove that if $\mathrm{SL}_{m}\left(R\left[X_{1}, X_{2}, \ldots, X_{n}\right]\right)=\mathrm{E}_{m}\left(R\left[X_{1}, X_{2}, \ldots, X_{n}\right]\right)$ for all $n, m \geq 0$ then all length four polynomial automorphisms of $R[X, Y]$ that are conjugates are stably tame.
\end{abstract}

\section{INTRODUCTION}

Through out this paper $R$ will be a UFD. Amongst the many unanswered questions about the the structure of $\mathrm{GA}_{2}(R)$, the group of polynomial automorphisms of the polynomial algebra $R[X, Y]$, stable tameness conjecture is a long standing one. In this paper we will prove that certain length four automorphisms are stably tame. We will also give an intriguing example of a length four automorphism which is length four and stably tame.

First we need a few definitions. A polynomial map is a map $F=\left(F_{1}, \ldots, F_{n}\right)$ : $\mathbb{A}_{R}^{n} \rightarrow \mathbb{A}_{R}^{n}$ where each $F_{i} \in R^{[n]}$. Such an $F$ is said to be invertible if there exists $G=\left(G_{1}, \ldots, G_{n}\right), G_{i} \in R^{[n]}$ such that $G_{i}\left(F_{1}, \ldots, F_{n}\right)=X_{i}$ for $1 \leq i \leq n$. The group of all polynomial automorphisms, $\operatorname{GA}_{n}(R)$ is defined as:

- $\mathrm{GA}_{n}(R)=\left\{F=\left(F_{1}, \ldots, F_{n}\right): F\right.$ is invertible $\}$.

An important goal in the study of polynomial automorphisms is to understand the structure of this group in terms of some of its well understood subgroups. An example of such a subgroup is

Tame subgroup: $\mathrm{T}_{n}(R)=\left\langle\operatorname{Af}_{n}(R), \mathrm{EA}_{n}(R)\right\rangle$ where

$$
\begin{array}{r}
\operatorname{Af}_{n}(R)=\left\{\left(a_{11} X_{1}+a_{12} X_{2}+\ldots+a_{1 n} X_{n}+b_{1}, \ldots, a_{n 1} X_{1}+. . a_{n n} X_{n}+b_{n}\right):\right. \\
\left.\left(a_{i j}\right) \in \mathrm{GL}_{n}(R) \text { and } b_{i} \in R\right\}
\end{array}
$$

is the subgroup of affine automorphisms of $\mathbb{A}_{R}^{n}$ and the elementary subgroup,

$$
\begin{array}{r}
\mathrm{EA}_{n}(R)=\left\{\left\langle\left(X_{1}, X_{2}, \ldots, X_{i-1}, X_{i}+f\left(X_{1}, \ldots, X_{i-1}, \hat{X}_{i}, X_{i+1}, \ldots, X_{n}\right), \ldots, X_{n}\right)\right\rangle:\right. \\
\left.f \in R\left[X_{1}, X_{2}, \ldots, \hat{X}_{i}, \ldots, X_{n}\right], i \in\{1, \ldots, n\}\right\}
\end{array}
$$

Another well studied subgroup of $\mathrm{GA}_{n}(R)$ is the triangular subgroup,

$$
\begin{array}{r}
\mathrm{BA}_{n}(R)=\left\{\left\langle\left(a_{1} X_{1}+f_{1}\left(X_{2}, \ldots, X_{n}\right), a_{2} X_{2}+f_{2}\left(X_{3}, \ldots, X_{n}\right), \ldots, a_{n} X_{n}+f_{n}\right)\right\rangle:\right. \\
\left.a_{i} \in R^{*}, f_{i} \in R\left[X_{i+1}, \ldots, X_{n}\right], 1 \leq i \leq n-1, f_{n} \in R\right\}
\end{array}
$$

If $R$ is a domain, then $\mathrm{GA}_{1}(R)=\mathrm{Af}_{1}(R)$. When $R$ is a field $k$ the following well known theorem gives us the structure of $\mathrm{GA}_{2}(k)$. Jun42, vdK53.

Theorem 1.1. (Jung, van der Kulk) If $k$ is a field then $\mathrm{GA}_{2}(k)=\mathrm{T}_{2}(k)$. Further, $\mathrm{T}_{2}(k)$ is the amalgamated free product of $\mathrm{Af}_{2}(k)$ and $\mathrm{BA}_{2}(k)$ over their intersection. 
A natural question that arises from Theorem 1.1 is whether $\mathrm{T}_{3}(k)$ is the whole group $\mathrm{GA}_{3}(k)$ ? Nagata Nag72 conjectured that the answer is no and gave a candidate counterexample.

Example 1.1. (Nagata)

$$
\text { Let } \begin{aligned}
F_{1} & =\left(X, Y+\frac{X^{2}}{t}, t\right) \text { and } F_{2}=\left(X+t^{2} Y, Y\right) \\
\text { Then } N & =F_{1}^{-1} \circ F_{2} \circ F_{1} . \\
& =\left(X+t\left(t Y+X^{2}\right), Y-2\left(t Y+X^{2}\right) X-t\left(t Y+X^{2}\right)^{2}, t\right) \in \mathrm{GA}_{3}(k)
\end{aligned}
$$

Using the following algorithrm from vdE00, we can conclude that $N \notin \mathrm{T}_{2}(k[t])$.

Let $F=(P(X, Y), Q(X, Y)) \in \mathrm{GA}_{2}(R)$ and $\operatorname{tdeg}(F)=\operatorname{deg}(P)+\operatorname{deg}(Q)$ and $h_{1}$ be the highest degree term of $P$ and $h_{2}$ that of $Q$.

Algorithrm 1.1. Input: $F=(P, Q)$.

1) Let $\left(d_{1}, d_{2}\right)=(\operatorname{deg}(P), \operatorname{deg}(Q))$.

2) If $d_{1}=d_{2}=1$, go to 7 .

3) If $d_{1} \neq d_{2}$, go to 5 .

4) If there exists $\tau \in \operatorname{Af}_{2}(R)$ with $\operatorname{tdeg}(\tau \circ F)<\operatorname{tdeg}(F)$, replace $F$ by $\tau \circ F$ and go to 1 , else stop : $\notin \mathrm{T}_{2}(R)$.

5) If $d_{2}<d_{1}$, replace $F$ by $(Q, P)$.

6) If $d_{1} \mid d_{2}$ and there exists $c \in R$ with $h_{2}=c h_{1}^{d_{2} / d_{1}}$, replace $F$ by $(X, Y-$ $\left.c X^{d_{2} / d_{1}}\right) \circ F \quad$ and go to 1 , else stop : $F \notin \mathrm{T}_{2}(R)$.

7) If $\operatorname{det} J F \in R^{*}$, stop: $F \in \mathrm{T}_{2}(R)$, else stop : $F \notin \mathrm{T}_{2}(R)$.

Shestakov and Umirbaev in 2002 SU03. proved that $N \notin \mathrm{T}_{3}(k)$ and thus proved Nagata's conjecture.

\section{Definition 1.1.}

Let $F, G \in \mathrm{GA}_{n}(R)$. Then

(1) $F$ is stably tame if there exists $m \in \mathbb{N}$ and new variables $X_{n+1}, \ldots, X_{n+m}$ such that the extended map $\widetilde{F}=\left(F, X_{n+1}, \ldots, X_{n+m}\right)$ is tame.

i.e $\left(F, X_{n+1}, \ldots, X_{n+m}\right) \in \mathrm{T}_{n+m}(R)$

(2) $F$ is tamely equivalent $(\sim)$ to $G$ if there exists $H_{1}, H_{2} \in \mathrm{T}_{n}(R)$ such that $H_{1} \circ F \circ H_{2}=G$.

(3) $F$ is stable tamely equivalent $\left(\sim_{\text {st }}\right)$ to $H \in \mathrm{GA}_{n+m}(R)$ if there exists $\widetilde{H_{1}}, \widetilde{H_{2}} \in$ $\mathrm{T}_{n+m}(R)$ such that $\widetilde{H_{1}} \circ \widetilde{F} \circ \widetilde{H}_{2}=H$ where $\widetilde{F}=\left(F, X_{n+1}, \ldots, X_{n+m}\right)$

Martha Smith proved Smi89 that $N$ from Nagata's example is stably tame with one more variable. This result led to the formulation of the following conjecture.

Conjecture 1. If $\mathrm{k}$ is a field and $F \in \mathrm{GA}_{n}(k)$ then $F$ is stably tame.

In her proof of the stable tameness of Nagata's example, Martha Smith exploited the decomposition of $N$ in Example 1.1 into certain special type of elementary automorphisms as shown in the example. This led to further study of such decompositions and the notion of the length of an automorphism, which we discuss below. The following proposition due to Wright is well known and a proof is given in Kut08. 
Proposition 1.1. Let $R$ be a domain $K$ its fraction field and $F \in \mathrm{GA}_{2}(R)$. Then $F=L \circ D_{a, 1} \circ F_{m} \circ F_{m-1} \circ \ldots \circ F_{1}$ where $L=(X+c, Y+d), D_{a, 1}=(a X, Y), F_{i}=$ $(X, Y+f(X))$ or $F_{i}=(X+g(Y), Y)$ for some $c, d \in R, a \in R^{*}, f(X), g(X) \in$ $K[X]$

\section{Definition 1.2.}

(1) Length of $F \in \mathrm{GA}_{2}^{0}(R)$ is the smallest natural number $\mathrm{m}$ such that $F=$ $D_{a, 1} \circ F_{m} \circ F_{m-1} \circ \ldots \circ F_{2} \circ F_{1}$ where each $F_{i}$ is either of the type $(X, Y+$ $\left.f_{i}(X)\right)$ or $\left(X+g_{i}(Y), Y\right)$ with $f_{i}(X), g_{i}(X) \in K[X], a \in R^{*}$ and $f_{i}(0)=$ $g_{i}(0)=0$.

(2) $\mathrm{L}^{(m)}(R)=\left\{F \in \mathrm{GA}_{2}^{0}(R): F\right.$ is of length $\left.m\right\}$

Remark 1.1. If $F \in \mathrm{L}^{(m)}(R)$ as above and $F=D_{a, 1} \circ F_{m} \circ F_{m-1} \circ \ldots \circ F_{2} \circ F_{1} \in$ $\mathrm{L}^{(m)}(R)$ then $F$ is tamely equivalent to $G=F_{m} \circ F_{m-1} \circ \ldots \circ F_{2} \circ F_{1}$. Thus $F$ is stably tame iff $G$ is stably tame.

It is easy to see that Nagata's example is of length three and it is stably tame with one more variable. Drensky and Yu [DY01] began a systematic study of length three automorphisms and proved the following result.

Theorem 1.2. (Drensky, Yu) Let $k$ be a field of characteristic zero and $F \in$ $L^{(3)}(k[t])$ such that $F=F_{1}^{-1} \circ G \circ F_{1}$ where $F_{1}=(X, Y+f(X)), G=(X+g(Y), Y)$ with $f(X), g(X) \in k[t][X]$. Then $F$ is stably tame with one more variable.

The following theorem was proved in $\operatorname{Kut08}$. Let $\mathrm{SL}_{n}(R)$ denote the set of all $n \times n$ matrices with entries from $\mathrm{R}$ and determinant equal to 1 and $\mathrm{E}_{n}(R)$ denote the group generated by the set of all nxn elementary matrices with entries from $R$.

Theorem 1.3. Suppose $R$ is a UFD such that $S L_{m}\left(R\left[X_{1}, X_{2}, \ldots, X_{n}\right]\right)=$ $E_{m}\left(R\left[X_{1}, X_{2}, \ldots, X_{n}\right]\right)$ for all $n, m \geq 0$. Then $F \in L^{(3)}(R) \Rightarrow F$ is stably tame.

This theorem was also claimed by Edo in [Edo05] without the assumption that $\mathrm{SL}_{m}\left(R\left[X_{1}, X_{2}, \ldots, X_{n}\right]\right)=$ $\mathrm{E}_{m}\left(R\left[X_{1}, X_{2}, \ldots, X_{n}\right]\right)$ for all $n, m \geq 0$. However, it is the author's contention that this assumption is required for the proof provided in Edo05 to hold. So a natural question at this point to ask is if $F \in \mathrm{L}^{(4)}(R)$ stably tame? As an evidence to an affirmative answer to this question, we prove the following theorem in this paper.

Theorem 1.4. (Main Theorem) Let $R$ be a UFD and $F \in L^{(4)}(R)$ and $F=$ $G_{1}^{-1} \circ F_{1}^{-1} \circ G_{1} \circ F_{1}$ where $F_{1}=(X, Y+f(X)), G_{1}=(X+g(Y), Y), f(X), g(X) \in$ $K[X]$ with $f(0)=g(0)=0$. Then $F$ is stably tame.

Remark 1.2. In BvdEW Berson,van den Essen and Wright recently proved that if $F \in \mathrm{GA}_{2}(R)$, where $R$ is a regular ring then $F$ is stably tame. This is a much stronger result. However, our result does not require the ring to be regular.

Before we present the proof of the main theorem, here is an example of a non tame automorphism of length four. 
Example 1.2. Let $R$ be a UFD and $t \in R \backslash\{0\}$.

Let $F_{1}=\left(X, Y+\frac{(t+1)^{3} X^{2}}{t}\right)$

$$
\begin{aligned}
G_{1}=\left(X+\frac{t^{2} Y}{(t+1)}\right) \text { and } \\
F=G_{1}^{-1} \circ F_{1}^{-1} \circ G_{1} \circ F_{1} \\
=\left(X+t(t+1) X^{2}-t^{5} Y^{2}-t^{3}(t+1)^{6} X^{4}-2 t^{3}(t+1) X Y-2 t^{2}(t+1)^{4} X^{3}\right. \\
\quad-2 t^{3}(t+1)^{4} X^{2} Y, Y-t^{3}(t+1) Y^{2}-t(t+1)^{7} X^{4} \\
\left.\quad-2 t(t+1)^{2} X Y-2(t+1)^{5} X^{3}-2 t(t+1)^{5} X^{2} Y\right)
\end{aligned}
$$

Using the algorithrm 1.1 we can see that $F \notin \mathrm{T}_{2}(R)$.

\section{Structure Of Length Four Automorphisms}

Lemma 2.1. Let $R$ be a U.F.D, $K$ its fraction field, and $A(X), B(X) \in R[X], b \in$ $R$ be such that $A(0)=B(0)=0, \operatorname{gcd}(B, b)=1$. Then $A\left(\frac{B(X)}{b}\right) \in R[X] \Rightarrow A(X)=$ $C(b X)$.

Proof.

$$
\begin{aligned}
A\left(\frac{B(X)}{b}\right) & =A\left(0+\frac{B(X)}{b}\right) \\
& =\sum_{i=0}^{n} A^{(i)}(0) \frac{B(X)^{i}}{i ! b^{i}}=\sum_{i=1}^{n} A^{(i)}(0) \frac{B(X)^{i}}{i ! b^{i}} \\
& =B(X) \sum_{i=1}^{n} A^{(i)}(0) \frac{B(X)^{i-1}}{i ! b^{i}} \in R[X]
\end{aligned}
$$

$\Rightarrow B(X) \sum_{i=0}^{n} A^{(i)}(0) b^{n-i} \frac{B(X)^{i-1}}{i !} \equiv 0 \bmod b^{n}$. Since $\operatorname{gcd}(B, b)=1$, we get

$$
\sum_{i=1}^{n} A^{(i)}(0) b^{n-i} \frac{B(X)^{i-1}}{i !} \equiv 0 \bmod b^{n}
$$

Putting $\mathrm{X}=0$ in $(*)$ gives us $A^{\prime}(0) \equiv 0$ modb. i.e Coefficient of $\mathrm{X}$ in $\mathrm{A}(\mathrm{X})$ is divisible by b. So $(*)$ becomes

$$
\left.\sum_{i=2}^{n} A^{(i)}(0) b^{n-i} \frac{B(X)^{i-1}}{i !}=B(X)\left(\sum_{i=2}^{n} A^{(i)}(0) b^{n-i} \frac{B(X)^{i-1}}{i !}\right) \equiv 0 \bmod b^{n}\right)
$$

Again $\operatorname{gcd}(B, b)=1$ gives us

$$
\sum_{i=2}^{n} A^{(i)}(0) b^{n-i} \frac{B(X)^{i-1}}{i !} \equiv 0 \quad \bmod b^{n}
$$

Putting $\mathrm{X}=0$ in $\left({ }^{* *}\right)$ we get, $\frac{A^{\prime \prime}(0)}{2 !} \equiv 0\left(\bmod b^{2}\right)$. i.e Coefficient of $X^{2}$ in $\mathrm{A}(\mathrm{X})$ is divisible by $b^{2}$. Proceeding like this one gets that for all $k \geq 1$ coefficient of $X^{k}$ in $\mathrm{A}(\mathrm{X})$ is divisible by $b^{k}$. 
Now we prove a lemma about the structure of automorphisms in $\mathrm{L}^{(4)}(R)$ where $R$ is a U.F.D. Let $F=G_{2} \circ F_{2} \circ G_{1} \circ F_{1} \in \mathrm{L}^{(4)}(R), F_{i}=\left(X, Y+\frac{A_{i}(X)}{a_{i}}\right), G_{i}=$ $\left(X+\frac{B_{i}(Y)}{b_{i}}, Y\right), A_{i}(X), B_{i}(X) \in R[X], a_{i}, b_{i} \in R$ and $A_{i}(0)=B_{i}(0)=0$ for $i=1,2, \operatorname{gcd}\left(A_{i}, a_{i}\right), \operatorname{gcd}\left(B_{i}, b_{i}\right)=1$.

Lemma 2.2. We use the same notations as above. Then $A_{2}(X)=C\left(b_{1} X\right)$ and $B_{1}(Y)=$ $D\left(a_{2} Y\right)$ for some $C(X), D(X) \in R[X]$ and $\operatorname{gcd}\left(a_{2}, b_{1}\right)=1$.

Proof.

$$
\begin{aligned}
F & =G_{2} \circ F_{2} \circ G_{1} \circ F_{1} \\
& =\left(X+\frac{B_{1}\left(Y+\frac{A_{1}(X)}{a_{1}}\right)}{b_{1}}+\frac{B_{2}\left(Y+\frac{A_{1}(X)}{a_{1}}+\frac{A_{2}\left(X+\frac{B_{1}\left(Y+\frac{A_{1}(X)}{a_{1}}\right)}{b_{1}}\right)}{a_{2}}\right)}{b_{2}},\right.
\end{aligned}
$$

$$
\left.Y+\frac{A_{1}(X)}{a_{1}}+A_{2}\left(X+\frac{B_{1}\left(Y+\frac{A_{1}(X)}{a_{1}}\right)}{b_{1}}\right)\right)
$$

Putting $\mathrm{X}=0$ in the second coordinate of $F$ we get $A_{2}\left(\frac{B_{1}(Y)}{b_{1}}\right) \in R[X]$. Then applying Lemma 2.1, we get $A_{2}(X)=C\left(b_{1} X\right)$. Similarly putting $Y=0$ in the first coordinate of $F^{-1}$ we get $B_{1}(Y)=D\left(a_{2} Y\right)$. Since $A_{2}(X)=C\left(b_{1} X\right)$, we know that $\operatorname{gcd}\left(C\left(b_{1} X\right), a_{2}\right)=1 \Rightarrow \operatorname{gcd}\left(a_{2}, b_{1}\right)=1$

2.1. Proof of the Main Theorem. Following useful lemma was proved in DY01] when $R=k[t]$ and was proved when $R$ is a UFD in [Kut08.

Lemma 2.3. Let $F=F_{1}^{-1} \circ G \circ F_{1} \in L^{(3)}(R)$ where $F_{1}=\left(X, Y+\frac{A_{1}(X)}{a}\right), G=$ $(X+g(Y), Y), A_{1}(X) \in R[X], g(Y) \in K[Y], a \in R$. Then $g(Y)=D(a Y)$ for $D(Y) \in$ $R[Y]$ and $a \mid D(Y)$.

Let $\left.F_{1}=\left(X, Y+\frac{A(X)}{a}\right), G_{1}=\left(X+\frac{B(Y)}{b}\right), Y\right)$ and $F=G_{1}^{-1} \circ F_{1}^{-1} \circ G_{1} \circ$ $F_{1}$ where $A(X) \in R[X], B(Y) \in R[Y], A(0)=B(0)=0$. We may assume that $\operatorname{gcd}(A(X, a))=\operatorname{gcd}(B(Y), b)=1$. By Lemma 4 we also know that $\operatorname{gcd}(a, b)=$ $1, A(X)=C(b X), B(Y)=D(a Y)$ for some $C(X) \in R[X], D(Y) \in R[Y]$, with $C(0)=D(0)=0$.

$$
\begin{gathered}
F=\left(X+\frac{D(a Y+C(b X))-D(a Y+C(b X)-C(b X+D(a Y+C(b X))))}{b},\right. \\
\left.Y+\frac{C(b X)-C(b X+D(a Y+C(b X)))}{a}\right) .
\end{gathered}
$$

Claim 2.1. $a \mid D(Y)$ 
Let $S=\left\{1, b, b^{2}, \ldots\right\}$ and $R_{b}=S^{-1} R$. Clearly $G_{1} \in \mathrm{GA}_{2}\left(R_{b}\right)$ and so $G_{1} \circ F \in$ $\mathrm{GA}_{2}\left(R_{b}\right)$.

$$
\begin{aligned}
G_{1} \circ F & =G_{1} \circ G_{1}^{-1} \circ F_{1}^{-1} \circ G_{1} \circ F_{1} \\
& =F_{1}^{-1} \circ G_{1} \circ F_{1} \\
& =\left(X+\frac{D(a Y+C(b X))}{b}, Y+\frac{C(b X)-C(b X+D(a Y+C(b X)))}{a}\right) \in \mathrm{L}^{(3)}\left(R_{b}\right)
\end{aligned}
$$

So by Lemma 2.3 we have that $a \mid \frac{D(Y)}{b}$ in $R_{b}$. Since $\operatorname{gcd}(a, b)=1$, this implies that $a \mid D(Y)$. Hence the claim. .

Notice that by the claim $a$ divides all the terms in the first coordinate of $F$ in 2 except $X$. So we have

$$
\begin{aligned}
F & =(X+a P(X, Y), Y+Q(X, Y)) \text { where } \\
P(X, Y) & =\frac{D(a Y+C(b X))-D(a Y+C(b X)-C(b X+D(a Y+C(b X))))}{a b} \text { and } \\
Q(X, Y) & =\frac{C(b X)-C(b X+D(a Y+C(b X)))}{a} .
\end{aligned}
$$

Let $E=(X+a W, Y, W)$ and $L=(X, Y-Q(X, 0), W-P(X, 0))$ then

$$
\begin{aligned}
F & \sim_{\mathbf{s t}}(X+a P(X, Y), Y+Q(X, Y), W) \\
& \sim F_{1}=(X+a P(X, Y), Y+Q(X, Y), W+P(X, Y)) \\
& \sim E \circ F_{1} \circ E^{-1}=(X, Y+Q(X+a W, Y), W+P(X+a W, Y)) . \\
& \sim F^{1}=L \circ E \circ F_{1} \circ E^{-1} \\
& =(X, Y+Q(X+a W, Y)-Q(X, 0), W+P(X+a W, Y)-P(X, 0)) \\
=(X, Y+ & \frac{C(b X+a b W)-C(b X+a b W+D(a Y+C(b X+a b W)))}{a}-\frac{C(b X)+C(b X+D(C(b X)))}{a}, \\
W+ & \frac{D(a Y+C(b X+a b W))-D(C(b X))}{a b} \\
-\frac{D(a Y+C(b X+a b W)-C(b X+a b W+D(a Y+C(b X+a b W))))}{a b} & \left.+\frac{D(C(b X)-C(b X+D(C(b X))))}{a b}\right)
\end{aligned}
$$


We can compute that $F^{1}=G_{2}^{1} \circ F_{2}^{1} \circ G_{1}^{1} \circ F_{1}^{1}$ where

$$
\begin{aligned}
F_{1}^{1} & =\left(X, Y+\frac{C(b(X+a W))-C(b X)}{a}, W\right), \\
G_{1}^{1} & =\left(X, Y, W+\frac{D(a Y+C(b X))-D(C(b X))}{a b}\right), \\
F_{2}^{1} & =\left(X, Y-\frac{C(b(X+a W)+D(C(b X)))+C(D(C(b X)))}{a}, W\right) \\
\text { and } G_{2}^{1} & =\left(X, Y, W-\frac{D(b Y+C(b X)-C(b X+D(C(b X))))}{a b}\right. \\
+\frac{D(C(b X)-C(b X+D(C(b X))))}{a b} &
\end{aligned}
$$

Since $F_{1}^{1} \in \mathrm{EA}_{2}(R[X]), F^{1}$ is tamely equivalent to $F^{1} \circ\left(F_{1}^{1}\right)^{-1} \in \mathrm{L}^{(3)}(R[X])$ and hence stably tame by Theorem 1.3 . Thus we get that $F$ is stably tame.

2.2. An intriguing Example. Using the notations above we give an example of a length four automorphism which is not a commutator. Further, in this example, $a \neq b$. However, this automorphism is stably tame!

Example 2.1. Let $R$ be a domain and $t \in R \backslash\{0\}$.

$$
\begin{aligned}
F_{1}= & \left(X, Y+\frac{X^{2}}{t}\right) \\
F_{2}= & (X, Y+(t-1) X) \\
G_{1}= & (X+(t+1) Y, Y) \\
G_{2}= & \left(X-\frac{Y^{2}}{t}, Y\right) \text { and } \\
F= & G_{2} \circ F_{2} \circ G_{1} \circ F_{1} \\
= & \left(X+(t+1) Y+3 X^{2}-t^{3} Y^{2}-t X^{2}-t X^{4}-2 t^{2} X Y\right. \\
& \quad+2 t X Y-2 t^{2} X^{2} Y-2 t X^{3}+2 X^{3}, \\
& \left.\quad t^{2} Y+(t-1) X+t X^{2}\right)
\end{aligned}
$$

Then $F \in \mathrm{L}^{(4)}(R)$. Using the Algorithrm 1.1 we get that $F \notin \mathrm{T}_{2}(R)$.

Let $P(X, Y)=(t+1) Y+3 X^{2}-t^{3} Y^{2}-t X^{2}-t X^{4}-2 t^{2} X Y+2 t X Y$

$$
-2 t^{2} X^{2} Y-2 t X^{3}+2 X^{3}
$$

$$
Q(X, Y)=t^{2} Y+(t-1) X+t X^{2}
$$

and $\widetilde{Q}(X, Y)=X+t Y+X^{2}$. 
We extend $F$ to $\widetilde{F}=(F, Z) \in \mathrm{GA}_{3}(R)$ and define the following elementary automorphisms of $R[X, Y, Z]$.

$$
\begin{aligned}
\tau & =(X, Y, W+\widetilde{Q}(X)) \\
\eta & =(X, Y-t Z, Z) \\
\phi & =(X-t Z, Y, Z)
\end{aligned}
$$

Also, let $\pi=(-Y, X, Z)$

Then $\pi \circ \eta \circ \widetilde{F} \circ \tau=(X+t Z, X+P(X, Y), Z+\widetilde{Q}(X, Y))$ and

$$
\begin{aligned}
& \widetilde{F^{1}}= \pi \circ \eta \circ F^{1} \circ \tau \circ \phi \\
&=(X, X-t Z+P(X-t Z, Y), Z+\widetilde{Q}(X-t Z, Y)) \\
&=\left(X, X-t Z+(t+1) Y+t^{3} Y^{2}+3(X-t Z)^{2}-t(X-t Z)^{2}\right. \\
&-t(X-t Z)^{4}-2 t^{2}(X-t Z) Y+2 t(X-t Z) Y-2 t^{2}(X-t Z)^{2} Y \\
&\left.-2 t(X-t Z)^{3}+2(X-t Z)^{3}, Z+(X-t Z)+t Y+(X-t Z)^{2}\right) \\
&=\left(X, Y+t Y+X-t Z+t^{3} Y^{2}+3(X-t Z)^{2}-t(X-t Z)^{2}-t(X-t Z)^{4}\right. \\
& \quad-2 t^{2}(X-t Z) Y+2 t(X-t Z) Y-2 t^{2}(X-t Z)^{2} Y \\
&\left.\quad-2 t(X-t Z)^{3}+2(X-t Z)^{3}, Z+(X-t Z)+t Y+(X-t Z)^{2}\right)
\end{aligned}
$$

Then $\widetilde{F^{1}}=\left(X, Y+P_{1}(X, Y, Z), Z+Q_{1}(X, Y, Z)\right)$ where

$$
P_{1}(X, Y, Z)=P(X-t Z, Y)-Y+X \text { and } Q_{1}(X, Y, Z)=\widetilde{Q}(X-t Z, Y)
$$

Notice that $\widetilde{F} \sim_{\text {st }} \widetilde{F^{1}}$. Let $\Theta=\left(X, Y-P_{1}(X, 0,0), Z-Q_{1}(X, 0,0)\right)$.

Then $\widetilde{F^{1}} \sim \Theta \circ \widetilde{F^{1}}$. Clearly the following automorphisms are in $\mathrm{BA}_{3}(R)$.

$$
\begin{aligned}
& \widetilde{F_{1}}=\left(X, Y+\frac{(X+t Z)+(X+t Z)^{2}-X-X^{2}}{t}, Z\right) \\
& \widetilde{G_{1}}=(X, Y, Z+t Y)
\end{aligned}
$$

Then we have that $\Theta \circ{\widetilde{F^{1}}}^{1}={\widetilde{F_{1}}}^{-1} \circ \widetilde{G_{1}} \circ \widetilde{F_{1}}$. So $\tau_{1} \circ \widetilde{F^{1}} \in \mathrm{T}_{3}(R)$. Hence $F$ is stably tame.

\section{REFERENCES}

[BvdEW] Joost Berson, Arno van den Essen, and David Wright. Stable tameness of two-dimensional polynomial automorphisms over a regular ring. Preprint, arXiv:0707.3151v5 [math.AC].

[DY01] Vesselin Drensky and Jie-Tai Yu. Tame and wild coordinates of $K[z][x, y]$. Trans. Amer. Math. Soc., 353(2):519-537, 2001.

[Edo05] Eric Edo. Totally stably tame variables. J. Algebra, 287(1):15-31, 2005.

[Jun42] Heinrich W. E. Jung. Über ganze birationale Transformationen der Ebene. J. Reine Angew. Math., 184:161-174, 1942.

[Kut08] Sooraj Kuttykrishnan. Some stably tame polynomial automorphisms. To appear in Journal Of Pure And Applied Algebra, 2008. 
[Nag72] Masayoshi Nagata. On automorphism group of $k[x, y]$. Kinokuniya Book-Store Co. Ltd., Tokyo, 1972. Department of Mathematics, Kyoto University, Lectures in Mathematics, No. 5 .

[Smi89] Martha K. Smith. Stably tame automorphisms. J. Pure Appl. Algebra, 58(2):209-212, 1989.

[SU03] Ivan P. Shestakov and Ualbai U. Umirbaev. The Nagata automorphism is wild. Proc. Natl. Acad. Sci. USA, 100(22):12561-12563 (electronic), 2003.

[vdE00] Arno van den Essen. Polynomial automorphisms and the Jacobian conjecture, volume 190 of Progress in Mathematics. Birkhäuser Verlag, Basel, 2000.

[vdK53] W. van der Kulk. On polynomial rings in two variables. Nieuw Arch. Wiskunde (3), 1:33-41, 1953.

E-mail address: sooraj@cse.wustl.edu

Department of Computer Science, Washington University in St. Louis 